\title{
UN ANÁLISIS DE LOS DETERMINANTES ECONOMICOS DE LA MIGRACIÓN MEXICANA LEGAL E ILEGAL HACIA LOS ESTADOS UNIDOS
}

\author{
Mario I. Blejer \\ HARRY G. JOHNSON \\ Arturo C. Porzecanski*
}

EL PROPósito de este documento es investigar las pautas generales de la migración mexicana, legal e ilegal, hacia los Estados Unidos y analizar empíricamente sus principales determinantes económicos. Antes que nada, se consideran los aspectos económicos del flujo migratorio en sí mismo, más que en relación con la estimación de los efectos a corto plazo o de las consecuencias a largo plazo de la migración mexicana a los Estados Unidos en las economías mexicana o americana. ${ }^{1}$ Sin embargo, se espera que el análisis de los determinantes económicos de la migración mexicana proporcione ciertas luces para la comprensión de las políticas involucradas con las implicaciones de este movimento migratorio.

\section{Patrones de la migración mexicana hacia los Estados Unidos}

En relación con lo que actualmente ocurre con el paso de la migración mexicana hacia los Estados Unidos, es usual poner de relieve, históricamente hablando, que tal migración ha sido insignificante. En realidad, en el periodo desde 1820 a 1880 no más de 25119 ciudadanos mexicanos llegaron a los Estados Unidos, número que representa exactamente $0.25 \%$ del total de inmigrantes, que fue de 10189429 . De manera similar, durante 1901-1950 llegaron 810841 mexicanos, los que

* Departamento de Economía, Universidad Hebrea de Jerusalem y Centro para el Desarrollo de Estudios sobre América Latina, Universidad de Bos en; Departamento de Economía, Universidad de Chicago, y Departamento de Investigación, Centro de Estudios Monetarios Latinoamericanos (CEMLA), respectivamente.

1 Esto ocurre debido a que la comparación y estimación de los costos y beneficios de tales movimientos migratorios requeriría el uso de datos detallados, los que al presente son inexactos o no están disponibles. Para un ejemplo de un intento reciente, aunque parcial de medir las consecuencias sobre la economía de México de la migración mexicana a Estados Unidos, véase el trabajo de Howard J. Campbell. 
constituían, más o menos, el $4 \%$ del número total de inmigrantes (estimado en 20201876 ). ${ }^{2}$

Durante el siglo veinte la migración mexicana ha tendido a fluctuar en lo que a primera vista parece ser una respuesta particular directa a las condiciones económicas en los Estados Unidos, dentro del contexto de una política tolerante de inmigración. Así, las cifras que se muestran en el cuadro 1 indican de manera exacta lo que quizás ocurrió a la proporción de migrantes mexicanos en ese total de inmigrantes. Por ejemplo, mientras en el primer decenio los mexicanos fueron $0.6 \%$ del total de inmigrantes, en el tercer decenio (1821-1930) esta proporción se elevó a $11.2 \%$ y disminuye de nuevo al $4.2 \%$ durante el cuarto decenio (19301940). ${ }^{3}$ Esto ha llevado a un analista a establecer que, históricamente, "los trabajadores mexicanos han sido cortejados en las épocas de escasez de mano de obra, pero han sido tratados como extranjeros indeseables cuando la escasez desaparece". 4

\section{Cuadro 1}

INMIGRACIÓN LEGAL MEXICANA A LOS EsTADOS UNIDOS

\begin{tabular}{ccc}
\hline Periodo do tiempo & Número de inmigrantes \\
\hline $1901-1910$ & 49642 \\
$1911-1920$ & & 219004 \\
$1921-1930$ & 459287 \\
$1931-1940$ & 2239 \\
$1941-1950$ & 60589 \\
$1961-1960$ & 299811 \\
1971 & 4530 & 537 \\
1972 & & 6403 \\
1973 & & 70141 \\
1974 & & 71586 \\
1975 & 62205 \\
\hline
\end{tabular}

FuENTE: Annual Report, Servicio de Inmigración y Naturalización de los Estados Unidos, diversos números.

Durante el periodo entre 1960 y 1975 , el número de inmigrantes legales de México ha tenđido a aumentar (véase el cuadro 2). Sin embargo, en pocos casos han tenido una importancia de consideración en la mano de obra de los Estados Unidos, porque una proporción considerable (entre 50 y $80 \%$ ) son dueños de casa, tienen hijos y otros dependientes. Además, más o menos un tercio de los inmigrantes legales provenientes de México, han sido exceptuados - a causa de sus vínculos familiares con un ciudadano americano- de las numerosas limitaciones existentes.

2 Obtenidos de varios números del Annual Report del Servicio de Inmigración y Naturalización de los Estados Unidos. No se ha considerado el período 1810-1890 porque no hay estadísticas de inmigración desde México para los años 1886-1893.

3 Éstos y los datos adicionales que se citan en todas partes son de los informes anuales del Servicio de Inmigración y Naturalización de los Estados Unidos.

4 Véase Walter A. Fogel, p. 44. 
Estas limitaciones existen, en efecto, desde mediados de 1968, cuando una enmienda al Acta sobre Inmigraciōn y Naturalización, de 1965, impuso un límite máximo de 120000 inmigrantes del Hemisferio Occidental por año sin límites para países determinados. ${ }^{5}$ Antes de 1968 no había una cuota formal de inmigrantes del Hemisferio Occidental; por eso, al menos en teoría, todos los mexicanos que deseaban inmigrar y podían cumplir ciertos prerrequisitos básicos, estaban en capacidad de hacerlo legalmente. Desde luego que siempre existieron dificultades individuales para la aplicación de estos requisitos básicos, la más importante de las cuales era la comprobación efectiva del solicitante de su autosuficiencia financiera, la cual muchas veces no la garantiza una simple oferta de trabajo. Los efectos de estos sistemas establecidos administrafivamente se aprecian a través de un incidente que tuvo lugar en julio de 1963, cuando, en respuesta a la creciente preocupación pública acerca del aumento de la inmigración desde el Hemisferio Occidental, el Departamento de trabajo de Estados Unidos anunció que en adelante, las ofertas de trabajo para los extranjeros mexicanos deberían ser "certificadas". Esta certificación implicaba verificar el empleo y, aún más importante, que el referido empteo no pudiera ser ocupado por trabajadores americanos o, a falta de esto, que los empleados extranjeros no fueran desfavorablemente afectados en el nivel de sus salarios nortéamericanos y en sus condiciones de trabajo. Este procedimiento, que estaba autorizado por el Acta de Inmigración y Naturalización de 1952, pero que fue habilitado en 1963 sólo en contra de los mexicanos, tuvo éxito en detener el crecimiento de la migración mexicana durante 1963, así como en provocar una reducción absoluta de la inmigración mexicana de su nivel de 1963 de 55253 al de 1964 con un número de 32 967. Fue sólo a mediados de 1971 que el monto de inmigrantes mexicanos recuperó su nivel de 1962 .

"Es claro que el interés principal en relación a la migración mexicana hacia los Estados Unidos está relacionado con el número creciente de inmigrantes ilegales. Mientras no hay estimaciones confiables acerca del número exacto de nacionales mexicanos que intentan o tienen éxito en ingresar ilegalmente a los Estados Unidos cada año -algunos dicen que más de dos millones-, es sabido que pese al magro y lento crecimiento de los recursos dedicados a su tarea, el Servicio de Inmigración y Naturalización (INS') ha localizado un número creciente de extranjeros mexicanos deportables ${ }^{6}$ (véase el cuadro 2 ).

Las razones para que un nacional mexicano intente el ingreso ilegal en vez del camino legal hacia los_Estados Unidos, son varias y algunas buenas. Primero, hay una razón histórica. En 1964 se terminó brusca-

5 Para el Hemisferio Oriental había una cuota global limitada de 170000 por año, pero no más de 20000 personas eran admitidas desde un mismo país.

6 David S. Norton y Marion F. Houstoun informan (p. 15) que mientras en 1964 se establecieron 1434 puestos de Patrullas de Agentes, en 1974 se establecieron 1718 (es decir, un $19.82 \%$ de aumento). 
Cuadro 2

INMIGRANTES LEGALES MEXICANOS A LOS ESTADOS UNIDOS Y EXTRANJEROS MEXICANOS DEPORTABLES, UBICADOS EN LOS ESTADOS UNIDOS

\begin{tabular}{|c|c|c|}
\hline $\boldsymbol{\phi}_{\bar{n}} \mathbf{n}$ & Innigranter legales & $\begin{array}{l}\text { Extranjeros } \\
\text { deportables } \\
\text { looglixados }\end{array}$ \\
\hline $\begin{array}{l}1960 \\
1961 \\
1962 \\
1963 \\
1964 \\
1965 \\
1966 \\
1967 \\
1968 \\
1969 \\
1970 \\
1971 \\
1972 \\
1973 \\
1974 \\
1975\end{array}$ & $\begin{array}{ll}32 & 684 \\
41 & 632 \\
55 & 291 \\
55 & 253 \\
32 & 967 \\
37 & 969 \\
45 & 163 \\
42 & 371 \\
43 & 563 \\
44 & 623 \\
44 & 469 \\
50.103 \\
64 & 040 \\
70 & 141 \\
71 & 586 \\
62 & 205\end{array}$ & $\begin{array}{rr}29 & 651 \\
29 & 817 \\
30 & 272 \\
39 & 124 \\
43 & 844 \\
55 & 349 \\
89 & 751 \\
108 & 327 \\
151705 \\
201 & 636 \\
277 & 377 \\
348 & 178 \\
430 & 213 \\
576 & 923 \\
709 & 959 \\
680 & 392\end{array}$ \\
\hline
\end{tabular}

Fuente: Annual Report, Servicio de Inmigración y Naturalización de los Estados Unidos, diversos números.

mente y en forma masiva el programa de trabajadores-visitantes -el famoso programa "bracero"- que en determinado momento llegó a emplear unos 200000 mexicanos por año. Hay indicios de que la mayoría de estos trabajadores (y sus familias) regresaron después ilegalmente a los Estados Unidos, simplemente porque no podían reubicarse en México, ni podían cumplir los requisitos recientes (mediados de 1963) bastante restrictivos para inmigrar a los Estados Unidos. Segundo, porque los trámites de la migración legal, desde mediados de 1968, tales como plazos prolongados para aquellos que no estaban comprendidos en la cuota del Hemisferio Occidental y para los inmigrantes potenciales, la dificultad de obtener documentos claves, el pago de varios estipendios extraordinarios, la aprobación de estrictos controles de salud y, la mayor dificultad de todas, la presentación de la prueba de autosuficiencia financiera. Por último, además de ser mucho más rápida y barata, la migración ilegal, aun cuando falle por causas de là deportación, aparece vinculada a un pequeño financiamiento o costo físico.

- Para el pasado, Wayne A. Cornelius y Juan Diez-Canedo señalan (p. 7), que en varias comunidades mexicanas estudiadas a través de encuestas y entrevistas, "una estructura de actitudes y normas de comportamiento se había desarrollado con fuerte apoyo a la migración a los Estados Unidos. Esto es una aceptada y altamente institucionalizada imagen de la vida de la comunidad. También... hay un pequeño estigma social, o bien no lo hay, unido a la migración ilegal a los Estados Unidos. Si alguien es capturado y deportado por el INS, ello es considerado simplemente como 'mala suerte'. Por otro lado, el que tiene éxito en evadir al INs, en especial después de múltiples estadías en los Estados Unidos, mejora su posición entre sus iguales". 


\section{Determinantes ECONÓMICOS DE.LA MigRACIÓN}

La contribución de los economistàs a la comprensión de la migración, se centra en el punto de vista de que ésta constituye un acto de inversión de capital humano; o sea, la expresión de una decisión racional basada en una evaluación de costos y beneficios de la migración $\mathrm{y}$, por lo tanto, de la tasa de beneficios existente.

Aun cuando no existen estudios económicos cuantitativos previos sobre la migración mexicana a los Estados Unidos, se han hecho algunas encuestas o verificaciones mediante análisis de regresión sobre la influencia de los factores económicos en la migración interna en México. Por ejemplo, Jonathan Kings elaboró un modelo muy simple de migración, con datos de todas las entidades federativas de México, obtenidos del censo de 1970, y encontró que, entre las diversas variables incluidas, la tasa de desempleo influía de manera importante y con más frecuencia que las variables salarios e ingresos. En otro estudio, basado en una encuesta de iñmigrantes a la ciudad industrial de Monterrey, H. Browning y W. Feindt concluyen que casi dos terceras partes de los inmigrantes llegaron por causas relacionadas con cuestiones de trabajo. ${ }^{9}$

Un estudio reciente de Wayne A. Cornelius y Juan Diez-Canedo, ${ }^{10}$ basado en entrevistas realizadas en nueve pequeñas comunidades en el estado de Jalisco, sobre el tema de la inmigración mexicana a los Estados Unidos, evidencia que "un exceso de población en relación con la extensión de la tierra cultivable y el número de oportunidades de empleo no agrícola, parece ser uno de los factores básicos que propician la inmigración en las comunidades estudiadas". Los autores deducen que, para muchos de los residentes entrevistados "la decisión de ir a los Estados Unidos es eminentemente racional, en términos de las diferencias económicas frente al propio trabajo, así como a la elevada probabilidad de encontrar un trabajo en los Estados Unidos".

Este estudio hace suponer que los mexicanos no son una excepción en cuanto al principio general de que las consideraciones económicas ejercen una influencia marcada en la decisión del migrante. No obstante, después de llevar a cabo una cuantificación de la capacidad explicativa de los factores económicos, es importante formular la siguiente pregunta: ¿qué probabilidad hay de que los factores económicos afectan tanto a la migración legal como a la ilegal? Hasta fechas recientes, poco se sabía sobre quiénes eran los inmigrantes ilegales y qué los caracterizaba 11 Pero un reciente estudio de David S. North y Marion F. Houstoun, basado en una encuesta de arrestados y extranjeros ilegales realizada en 1975, proporciona alguna información relevante para este problema. Mientras los

8 Véase Jonathan King, "Redistribución óptima del ingreso", Demografía y Economía, Vol. XI, Núm. 1, 1977.

- 9 Véase también el interesante trabajo de Unikel, Ruiz Chiapetto y Lazcano.

10 Cornelius y Diez-Canedo, p. 6.

11 Para un interesante y reciente ensayo véase el libro de Julián Samora. 
inmigrantes ilegales mexicanos son mayoritariamente hombres y por lo común no llevan con ellos a sus familiares, es interesante observar que varias características comunes fácil de apreciarse, acerca de su sensibilidad ante las variables económicas ( $v . g r$. antecedentes ocupacionales, edad y educación formal) no resultan muy diferentes de los de sus contrapartes legales (véase el cuadro 3). Esto lleva a suponer que si las consideraciones económicas - y en especial las relativas al trabajo- juegan un papel importante en la determinación de los flujos migratorios, tales consideraciones pueden de igual manera afectar y no de manera muy diferente, tanto a los migrantes legales como a los ilegales.

Cuadro 3

EDAD Y OCUPACIÓN ANTERIOR DE LOS INMIGRANTAS MEXICANOS EN 1975

\begin{tabular}{|c|c|c|}
\hline Caracteristicas & $\begin{array}{r}\text { Inmigrantes } \\
\text { legaies }(X)\end{array}$ & $\begin{array}{l}\text { Inmigranteg } \\
\text { ilegaled }(x)\end{array}$ \\
\hline $\begin{array}{l}\text { Grupos de odad } \\
16-24 \\
25-44 \\
45 y \text { más }\end{array}$ & $\begin{array}{r}39.2 \\
51.3 \\
9.6\end{array}$ & $\begin{array}{r}46.6 \\
47.2 \\
6.2\end{array}$ \\
\hline $\begin{array}{l}\text { Antecedentes ocupacionales } \\
\text { Trabajadores calificados } \\
\text { Trabajadores seqioalifioados } \\
\text { Trabajadores no calificados }\end{array}$ & $\begin{array}{r}16.9 \\
17.9 \\
65.1\end{array}$ & $\begin{array}{l}18.2 \\
24.8 \\
56.9\end{array}$ \\
\hline
\end{tabular}

FUeNTE: Para los migrantes legales, Anual Report, 1975 Servicio de Inmigración y Naturalización de los Estados Unidos; para"Ios migrantes ilegales, "Las características y el rol de los extranjeros ilegales en el mercado de investigación inédito, Administración del empleo y adiestramiento. Departamento de Trabajo de los Estados Unidos", D. S. North y M. F. Houstoun, documento de investigación inédito, Administración del empleo y adiestramiento, Departamento de Trabajo de los Estados Unidos, 1976.

a Solamente hombres para el grupo legales y $93 \%$ de hombres para el grupo ilegales, calculados mediante el mismo procedimiento al empleado por North y Houstoun, cuadro IV 2.

b Ocupaciones anteriores a la inmigración (los individuos sin ocupación anterior fueron eliminados): "calificados" incluye a profesionales, técnicos, artesanos y trabajadores similares. así como a propietarios, gerentes y administradores; "semicalificados" incluye vendedores, oficinistas y. trabajadores similares, operarios y agricultores, y "no calificados" incluye trabajadores agrícolas y no agrícolas, así como trabajadores del servicio doméstico.

\section{RESUltados EMPÍRICOS}

Con el propósito de realizar un análisis empírico, se cuantificó el flujo de la migración mexicana a los Estados Unidos mediante tres diferentes variables: el número de inmigrantes legales (LEG), el número de inmigrantes ilegales (ILEG) y el número total de inmigrantes (TOT $=L E G+$ ILEG). ${ }^{12}$ Por carecer de un indicador alternativo de los inmigrantes ilegales,

12 Se probó también una variable que mide el número de inmigrantes legales que se esperaba se agregaran a la mano de obra de los Estados Unidos (definida como 
y dado el interés de cuantificar las variaciones más que precisar el tamaño de tales flujos migratorios, se utilizaron las cifras de los extranjeros mexicanos deportables localizados por el INS, para construir nuestras series de ILEG. Una descripción detallada de todos los datos utilizados y sus respectivas fuentes, aparece en el apéndice $A$.

La hipótesis sujeta a prueba es que el número de migrantes de cada tipo es principalmente una función de dos variables económicas: a) la probabilidad de encontrar empleo en los Estados Unidos en relación con la probabilidad de encontrar empleo en México, y $b$ ) los beneficios diferenciales esperados del trabajo.

Se utilizaron varias medidas como aproximación de las variables $a$ ) y $b$ ). En los cuadros 4, 5 y 6 se muestran los resultados obtenidos para LEG, ILEG y TOT, respectivamente, cuando los diferenciales en las expectativas de encontrar trabajo en los Estados Unidos son medidas por la relación entre la tasa mexicana y la tasa americana de desempleo (Umx/Uus) y las diferencias de los beneficios esperados del trabajo se cuantificaron por medidas alternativas de los diferenciales del salario real (Wus/Wmx).

\section{Cuadro 4}

Determinantes económicos de la MigRación legal mexicana A LOS ESTADOS UNIDOS

\begin{tabular}{|c|c|c|c|c|c|}
\hline Periódo & 0 & $=1$ & 2 & $a_{3}$ & $\overline{\bar{R}}^{2} / F$ \\
\hline $1960-1974$ & $\begin{array}{c}10.43 \\
(78.02)\end{array}$ & $\begin{array}{l}0.26 \\
(2.70)\end{array}$ & & & $0.31 / 7.30$ \\
\hline $1960-1974$ & $\begin{array}{c}10.43 \\
(80.31)\end{array}$ & $\begin{array}{l}0.49 \\
(2.53)\end{array}$ & $\begin{array}{l}1.29 \\
(1.34)\end{array}$ & & $0.35 / 4.76$ \\
\hline $1960-1972$ & $\begin{array}{l}10.49 \\
(86.24)\end{array}$ & $\begin{array}{l}0.30 \\
(1.78)\end{array}$ & & $\begin{array}{c}0.70 \\
(0.89)\end{array}$ & $0.16 / 2.14$ \\
\hline
\end{tabular}

Estimados conforme a las ecuaciones siguientes:

1) $\log \mathrm{LEG}=a_{0}+a_{1} \log (U m x /$ Uus $)+u_{1}$

2) $\log$ LEG $=a_{0}+a_{1} \log (U m x /$ Uus $)+a_{2} \log (W u s / W m x) I+u$.

3) $\log$ LEG $=a_{0}+a_{1} \log (U m x / U u s)+a_{3} \log (W u s / W m x) A+u_{3}$

en donde: LEG = inmigración legal; $U m x=$ tasa de desempleo en México; Uus $=$ tasa de desempleo en los Estados Unidos; $W u s$ = tasa de salarios en los Estados Unidos; $W m x=$ tasa de salarios en México; $I=$ sector industrial; $A=$ sector agrícola; $R=$ coeficiente de determinación ajustado; $F=$ estadístico $F$; los valores entre paréntesis corresponden al estadístico $t$.

Los resultados obtenidos indican que los diferenciales internacionales de desempleo fluctúan entre el 16 y el $35 \%$ de las variaciones en el número de migrantes legales; entre el 71 y el $72 \%$ de la variación en el nú-

LEG, menos los clasificados como sin ocupación. Según esos resultados, no había diferencias sustanciales entre ellos y los LEG, por lo cual se decidió no consignarlos en detalle. 


\section{Cuadro 5}

Determinantes económicos DE La MIGRaCión ILEGaL a LOS EsTados UNIDOS

\begin{tabular}{|c|c|c|c|c|c|}
\hline Perfodo & a. & $a_{9}$ & $\dot{\theta}_{2}$ & $a_{3}$ & $\overline{\mathrm{R}}^{2} / \mathrm{s}$ \\
\hline $1960-1974$ & $\begin{array}{r}9.46 \\
(23.39)\end{array}$ & $\begin{array}{c}1.76 \\
(5.96)\end{array}$ & & & $0.71 / 35.55$ \\
\hline $1960-1974$ & $\begin{array}{c}9.47 \\
(23.53)\end{array}$ & $\begin{array}{c}1.20 \\
(2.01)\end{array}$ & $\begin{array}{c}-3.19 \\
(1.07)\end{array}$ & & $0.71 / 18.54$ \\
\hline $1960-1972$ & $\begin{array}{c}9.67 \\
(27.15)\end{array}$ & $\begin{array}{c}0.92 \\
(1.87)\end{array}$ & & $\begin{array}{l}-3.31 \\
(1.44)\end{array}$ & $0.72 / 16.05$ \\
\hline
\end{tabular}

Estimados conforme a las ecuaciones siguientes:

1): $\log \operatorname{ILEG}=a_{0}+a_{1} \log ($ Umx/Uus $)+u_{1}$

2): $\log$ ILEG $=a_{0}+a_{1} \log (U m x / U u s)+a_{2} \log (W u s / W m x) \mathrm{I}+u_{2}$

3): $\log$ ILEG $=a_{0}+a_{1} \log (U m x /$ Uus $)+a_{3} \log (W u s / W m x) A+u_{3}$

En donde: ILEG = inmigrantes ilegales; los símbolos restantes tienen el mismo significado que en el cuadro 4.

\section{Cuadro 6}

Determinantes económicos de la migración meXicana A LOS ESTADOS UNIDOS ${ }^{a}$

\begin{tabular}{|c|c|c|c|c|c|}
\hline Perlodo & $a_{0}$ & $a_{1}$ & $a_{2}$ & $a_{3}$ & $\overline{\mathrm{R}}^{2} / \mathrm{F}$ \\
\hline $1960-1974$ & $\begin{array}{c}10.50 \\
(32.66)\end{array}$ & $\begin{array}{c}1.28 \\
(5.46)\end{array}$ & & & $0.67 / 29.78$ \\
\hline $1960-1974$ & $\begin{array}{l}10.50 \\
(31.76)\end{array}$ & $(2.05)$ & $\begin{array}{l}-1.31 \\
(0.53)\end{array}$ & & $0.65 / 14.21$ \\
\hline $1960-1972$ & $\begin{array}{c}10.68 \\
(38.89)\end{array}$ & $\begin{array}{l}0.74 \\
(3.95)\end{array}$ & & $\begin{array}{c}-1.76 \\
(0.99)\end{array}$ & $0.56 / 12.76$ \\
\hline
\end{tabular}

a Estimados conforme a las ecuaciones siguientes:

1) $\log$ тот $=a_{0}+a_{1} \log (U m x / U u s)+u_{1}$

2) $\log$ тот $=a_{0}+a_{1} \log (U m x / U u s)+a_{2} \log (W u s / W m x) I+u_{2}$

3) $\log$ тот $=a_{0}+a_{1} \log (U m x / U u s)+a_{3} \log (W u s / W m x) A+u_{3}$

En donde тот $=$ total de inmigrantes; los símbolos restantes tienen el mismo significado que en el cuadro 4.

mero de migrantes ilegales, y entre el 65 y $67 \%$ de la variación en el número total de migrantes. Los diferenciales internacionales de salarios reales en dos sectores clave (industria y agricultura) no aumentan de manera significativa el nivel de la explicación estadística. Se consideraron también los diferenciales de salarios en otros sectores de la economía así como los salarios mínimos, sin gran mejoría en los resultados. En los casos de la migración ilegal y total, los coeficientes de los diferenciales de salarios son no significativos estadísticamente, diferentes de cero y tienden a registrar signo negativo. 
Una variable adicional independiente probada fue la de los diferenciales de salarios entre los países cuando el salario nominal esperado en lós Estados Unidos es deflacionado con el nivel de precios mexicanos (en vez del de Estados Unidos'). Esto se ha hecho por la sospecha de que muchos inmigrantes mexicanos, y en especial los ilegales, llegan a los Estados Unidos temporalmente con el objeto de acumular fondos y regresar con ellos a México (esta hipótesis es anticipada por Cornelius y Diez-Canedo). En este caso, los migrantes estarían interesados en el vaor real de sus salarios en términos de poder adquisitivo en México más que en el poder adquisitivo en Estados Unidos. Sin embargo, los resultados obtenidos con esta variable son menos satisfactorios que los consignados en los cuadros 4,5 y 6 , tanto en términos de la significación estadística de los coeficientes estimados como del nivel total de capacidad de explicación, en la parte de las variables independientes.

Se formuló y verificó también una hipótesis algo diferente cuyos resultados aparecen en el cuadro 7. La hipótesis es que el número de inmigrantes (LEG, ILEG y TOT) es una función de: a) el exceso de oferta de mano de obra en México, y $b$ ) la probabilidăd de obtener un salario en dólares.

\section{Cuadro 7}

DETERMINANTES ECONÓMICOS DE LA MIGRACIÓN MEXICANA A LOS Estados UNIDOS: RESUltados ADICIONALES ${ }^{a}$

\begin{tabular}{|c|c|c|c|c|c|}
\hline $\begin{array}{l}\text { Variable } \\
\text { dependiente }\end{array}$ & Perfodo & 。 & $a_{1}$ & $a_{2}$ & $\overline{\mathrm{B}}^{2} / \mathrm{F}$ \\
\hline \multirow[t]{2}{*}{ LEG } & $1960-1974$ & $\begin{array}{c}8.56 \\
(16.80)\end{array}$ & $\begin{array}{r}0.38 \\
(4.36)\end{array}$ & & $0.56 / 13.97$ \\
\hline & & $\begin{array}{c}8.38 \\
(99.54)\end{array}$ & $\begin{array}{c}0.43 \\
(5.71)\end{array}$ & $\begin{array}{l}-0.36 \\
(0.61)\end{array}$ & $0.70 / 17.14$ \\
\hline \multirow[t]{2}{*}{ IIEG } & $1960-1974$ & $\begin{array}{l}-0.58 \\
(0.38)\end{array}$ & $\begin{array}{c}2.13 \\
(8.10)\end{array}$ & & $0.82 / 65.55$ \\
\hline & & $\begin{array}{l}-0.64 \\
(0.40)\end{array}$ & $\begin{array}{l}2.15 \\
(7.62)\end{array}$ & $\begin{array}{l}-0.10 \\
(0.20)\end{array}$ & $0.81 / 30.38$ \\
\hline \multirow[t]{2}{*}{ Tor } & $1960-1974$ & $\begin{array}{c}2.83 \\
(2.71)\end{array}$ & $\begin{array}{c}1.62 \\
(8.93)\end{array}$ & & $0.85 / 79.77$ \\
\hline & & $\begin{array}{c}2.63 \\
(2.53)\end{array}$ & $\begin{array}{c}1.67 \\
(9.10)\end{array}$ & $\begin{array}{l}-0.40 \\
(1.20)\end{array}$ & $0.85 / 41.92$ \\
\hline
\end{tabular}

a Estimados conforme a las ecuaciones siguientes:

1) $\log$ LEG, $\log$ ILEG O $\log$ TOT $=a_{0}+a_{1} \log U m x+u_{1}$

2) $\log$ LEG, $\log$ ILEG O $\log$ TOT $=a_{0}+a_{1} \log U m x+a_{2}(W m s / U u s)+u_{2}$ restantes tienen el mismo significado que en el cuadro 4.

En donde $\frac{m}{W}=$ tasa real de salario mínimo en Estados Unidos; los símbolos restantes tienen el mismo significado que en el cuadro 4.

El exceso de oferta de mano de obra en México se estimó con la tasa de desempleo (Umx) y se cuantificé el salario alcanzable en Estados Unidos mediante la división del salario mínimo en términos reales por la tasa de desempleo en dicho país (es decir, $W_{u s}^{m} / U u s$ ). Los resultados 
son similares a los que se muestran en los cuadros 4 a 6: la tasa de desempleo en México puede explicar por sí misma entre el 56 y el $85 \%$ de la variación en las tres medidas del flujo migratorio, mientras que la suma de la variable del salario alcanzable no añade casi nada al poder explicativo de las ecuaciones porque carece de significación estadística. ${ }^{13}$

Dada la falta de confiabilidad en los datos mexicanos sobre desempleo, como erá de importancia crítica contar con una variable explicativa, se estimó (como se indica en el apéndice A) una segunda serie de tiempo mucho más amplia de Umx y se utilizó como una alternativa en todas las verificaciones previamente consideradas. Las estimaciones resultantes, un pequeño ejemplo de las cuales aparece en el apéndice B; resultaron ser muy similares en sus implicaciones.

\section{RESUMEN DE LOS HALlazgos E IMPliCACIONES DE POLÍTICA}

El hallazgo principal de nuestro análisis econométrico puede ser condensado en los siguientes cinco puntos:

1) Parece que los determinantes económicos de la inmigración legal, ilegal y totat mexieana a los Estados Unidos son en esencia tós mismos, pero la magnitud de las consecuencias de un cambio en la proporción de un determinante económico dado es muy diferente, en función de cuál sea el flujo migratorio que se considere;

2) Sin duda la más importante variable afectada por el número de inmigrantes legales, ilegales y en conjunto es el diferencial en la tasa de desempleo entre México y los Estados Unidos, y en especial la tasa de desempleo en México. Estos resultados son ampliamente consistentes con los obtenidos por otros investigadores que han estudiado los determinantes de la migración interna en México;

3) La elasticidad de la migración respecto a los diferenciales de desempleo entre países, es mucho más alta para los inmigrantes ilegales que para los legales; lo mismo cuenta para la elasticidad de la migración con respecto a la tasa de desempleo en México;

4) Los diferenciales de salarios reales, aun cuando son estimados, no parecen influir en el numero de inmigrantes legales, ilegales y totales,

5) El intento de cuantificar la probabilidad de un migrante de encontrar a la vez un empleo y obtener un salario americano, así como de que encontraran trabajo y obtuvieran el diferencial existente de salarios, fue infructuoso, pues los flujos migratorios continúan siendo determinados de manera primordial por consideraciones de desempleo.

Se encontró que existen tres importantes implicaciones políticas que pueden ser diseñadas con nuestros resultados estadísticos:

13 Se consideró también la probabilidad de obtener el diferencial de salarios, en vez del salario real mínimo en los Estados Unidos, aunque con resultados similares. 
(1) El hecho de que tanto los inmigrantes legales como ilegales estén afectados por las mismas variables económicas sugiere que la divisiỏn entre ambos tipos es esencialmente artificial y que es determinada de manera principal por las leyes de inmigración de los Estados Unidos. Así, mientras más clemente fuera la ley (en términos de su aceptación por los mexicanos que quieren migrar), mayor sería la proporción de migrantes legales frente a los ilegales; mientras más restrictiva la ley, la proporción mayor sería de inmigrantes ilegales. Sin embargo, el volumen del flujo migratorio como un todo está básicamente en función de aspectos económicos más que de consideraciones legales;

2) El hecho de que los inmigrantes ilegales sean mucho más sensibles a los cambios en las variabtes economicas que los inmigrantes legales, significa que una mejoría en las condiciones básicas de la economía mexicana, en relàción con ta đe los Estados Unídos, habría influido mucho más de lo informado en el flujo migratorio del pasado que en el actual. La diferencia observada en la mencionada sensibilidad, tiene un sentido relevante:" mientras el grueso de los inmigrantes legales de México (es decir aquellos no privilegiados por la cuota) deben esperar más de dos años para obtener una visa, ${ }^{14}$ el saldo (es decir los que están incluidos en la cuota), Ios que tienen lazos familiares, no tienen que hacerlo; lo cual rebaja su sensibilidad respecto a las consideraciones económicas, pues los inmigrantes ilegales están inclinados a responder mucho más rápidamente a los cambios en que los factores económicos porque están libres de restricciones institucionales; ${ }^{15}$

3) El hecho de que los diferenciales de salarios entre países no muestren ser estadísticamente significativos en la determinación de los flujos migratorios, no debe ser considerado como que son irrelevantes. Es probable que dichos diferenciales son relevantes para los migrantes potenciales, pero que no han sido estimados de manera adecuada en este trabajo. En realidad, durante el periodo analizado, los diferenciales de salarios existentes entre los países disminuyen debido a la política cambiaria de México: a despecho de un erecimento más rápido de los salarios y de una tasa más alta de inflación en relación a la de Estados Unidos durante los años de 1970, México no devaluó su moneda hasta el 10. de septiembre de 1976. Pero lo inevitable de la devaluación fue discutido en México desde varilos años antes de que se adoptara la medida y creemos

14 Por ejemplo, en enero de 1976, la Embajada de los Estados Unidos en la ciudad de México concedió visas de inmigrantes a aquellos solicitantes desde una fecha anterior al 15 de agosto de 1973 - un periodo de espera de por lo menos veintinueve meses. Esta información fue amablemente proporcionada por la señorita Donna $\mathrm{J}$. Hamilton del Consulado de los Estados Unidos en la ciudad de México.

${ }_{15}$ Sin embargo, el tiempo que se tarda en obtener una visa no es tan relevante en la decisión entre migración legal e ilegal como pareciera. No obstante ser contrario a la ley de inmigración de los Estados Unidos, un gran número de solicitantes de visas de inmigración cree firmemente que puede pasar el tiempo de espera en los Estados Unidos. Un funcionario del Consulado de los Estados Unidos en la ciudad de México informó que alrededor del $90 \%$ de los solicitantes que iniciaron su período de espera en enero de 1976 tenían domicilio en los Estados Unidos. 
que eso condujo a los migrantes potenciales a dar poca importancia a la reducción observada en los diferenciales de salarios entre los dos países, en espera de que la futura devaluación los restableciera (si no los aumentaba). En relación con esto, la variable que debería haberse utilizado - que hubiéramos querido estimar-es una que incluye las expectativas de ùna ganancia en el potencial del salario en dólares o, lo qừe es lo mismo, una que incluya un descuento en el potencial del salario en pesos.

En relación con esto, podría ser útil especular sobre las consecuencias de la reciente y abultada devaluación monetaria en México, sobre la salida de migrantes mexicanos. Se consideera que esta devalución debe haber tenido dos efectos: uno en el remanente de mexicanos radicados en los Estados Unidós y otro, en el flujo de mexicanos hacia ese país. En relación al primero, si bien es verdad que una gran proporción de la población mexicana ilegal en los Estados Unidos reside allí sólo temporalmente con el objeto de acumular fondos que capaciten a sus integrantes para lograr una mejor posicion económica luego de su regreso a México, entonces este aumento único en el valor de sus ahorros en dólares (en términos de pesos mexicanos) podría inducir a un cierto número a regresar a México más pronto de lo que tenían planeado. En cuanto al otro efecto, y si los migrantes mexicanos responden a los diferenciales de salarios, entonces la devaluación obviamente debería incrementar el flujo de migrantes legales e ilegales, porque se habría producido un importante aumento en los diferenciales de salarios reales, en relación con los de la predevaluación.

Sin embargo, los efectos de la devaluación sobre el flujo de migrantes podría dejar de materializarse como consecuencia de los recientes cambios en la legislación de los Estados Unidos, orientada a imponer duras penas a los empleadores que ocupen a extranjeros ilegales. En verdad, estas penas forzarían a los empleadores a considerar un coeficiente de riesgo al emplear nacionales mexicanos ilegales, y ello deprimiría tanto sus actuales como sus futuras expectativas de compensación. En este momento, no se dispone de información suficiente para determinar la magnitud de los efectos de la devaluación y del cambio de la legislación.

Lo que se espera que este documento aclare es que, dada la importancia de los diferenciales del desempleo como determinantes de ta migración mexicana a los Estados Unidos, las modificaciones en la tasa de cambio de México o en la legislación americana, no alterarán de manera sustancial las tendencias pasadas de la migración. La clave para disminuir el flujo de migración mexicana - si ello va a convertirse en un objetivo a largo plazo de la política de los Estados Unidos- está unido a la reducción de la brecha existente entre las oportunidades económicas en México y en los Estados Unidos. 


\section{APÉNDICE A}

\section{Descripción y fuente de los datos}

Los datos sobre el número de migrantes legales, exceptuando aquellos migrantes legales que figuran en la categoría "sin ocupación"; migrantes ilegales (definidos como extranjeros mexicanos deportables localizados), y migrantes totales (legales más ilegales), se obtuvieron de diversos números del Annual Report del Servicio de Inmigración y Naturalización de los Estados Unidos.

Los datos de la tasa de desempleo en los Estados Unidos se obtuvieron de varias ediciones del Economic Report of the President y del Survey of Current Business.

La primera serie de la tasa de desempleo en México (es decir, la empledada para los resultados que se proporcionan en el texto') fue calculada y proporcionada por el grupo de economistas del Centro de Estudios Económicos del Sector Privado, de México. El desempleo se estimó con base en una ecuación en la que el crecimento del empleo es una función positiva del crecimiento real del producto ponderado por los niveles de crecimiento de seis sectores claves de la economía, suponiendo una relación trabajo/producto constante. Los datos necesarios para calcular los parámetros relevantes fueron obtenidos de los censos de población de 1950 y 1960, como también del Banco de México.

La segunda serie de las tasas de desempleo en México (es decir, las usadas para los resultados proporcionados en el Apéndice B'). fue calculada por los autores. El desempleo se estimó mediante una ecuación en la cual la tasa de desempleo aparece como una función inversa de la tasa de crecimiento real del producto y como una función positiva de la razón capital/trabajo. Los datos necesarios para calcular los parámetros relevantes fueron obtenidos de los censos (sobre desempleo) de 1950 y 1970; de diversas ediciones del Informe Anual del Banco de México (para el crecimiento real del producto); de Cuentas nacionales y acervos de capital, 1950-1967 (México, Banco de México, 1969) y del International Financial Statistics (para el saldo de capital), y de datos inéditos, proporcionados por Nacional Financiera (para la población económicamente activa).

Datos sobre los salarios nominales en la industria, agricultura y otros sectores se obtuvieron de diversas ediciones del Yearbook of Labor Statistics de la Oficina Internacional del Trabajo. Estos fueron ajustados por el respectivo índice de precios al consumidor de los países: para los Estados Unidos obtenidos en diversas ediciones del Economic Report of the President y Survey of Current Business; para México, de la Nacional Financiera Estadísticas de la economía mexicana, y del Banco de México, Indicadores económicos.

Por último, los datos sobre salarios mínimos fueron obtenidos, para los 
Estados Unidos, de la información proporcionada por la Oficina de Estadísticas del Trabajo del Departamento del Trabajo de los Estados Unidos y, para México, de la información proporcionada por Nacional Financiera.

\section{Apéndice B}

DETERMINANTES ECONÓMICOS DE LA MIGRACIÓN MEXICANA A LOS Estados UNIDOS: RESULTADOS ADICIONALES SELECCIONADOS ${ }^{a}$

\begin{tabular}{|c|c|c|c|c|c|}
\hline $\begin{array}{l}\text { Variablo } \\
\text { dependionte }\end{array}$ & Período & $a_{0}$ & $a_{1}$. & $a_{2}$ & $\overline{\mathrm{R}}^{2} / \mathrm{F}$ \\
\hline \multirow[t]{2}{*}{ LEG } & $1950-1975$ & $\begin{array}{l}3.79 \\
2.40\end{array}$ & $\begin{array}{l}1.39 \\
(4.25)\end{array}$ & & $0.41 / 18.07$ \\
\hline & & $\begin{array}{c}10.27 \\
(54.86)\end{array}$ & & $\begin{array}{c}0.57 \\
(1.55)\end{array}$ & $0.05 / 2.41$ \\
\hline \multirow[t]{2}{*}{ ILEG } & $1957-1975$ & $\begin{array}{l}-9.55 \\
(5.05)\end{array}$ & $\begin{array}{l}4.26 \\
(11.19)\end{array}$ & & $0.87 / 125.33$ \\
\hline & & $\begin{array}{l}(10.57 \\
(31.97)\end{array}$ & & $\begin{array}{c}2.38 \\
(3.91)\end{array}$ & $0.44 / 15.26$ \\
\hline \multirow[t]{2}{*}{ TOT } & $1957-1975$ & $\begin{array}{l}-4.12 \\
(2.63)\end{array}$ & $\left(\begin{array}{c}3.25 \\
(10.34)\end{array}\right.$ & & $0.85 / 106.96$ \\
\hline & & $\begin{array}{l}11.29 \\
(42.13)\end{array}$ & & $\begin{array}{c}1.73 \\
(3.50)\end{array}$ & $0.38 / 12.25$ \\
\hline
\end{tabular}

a Estimados conforme a las ecuaciones siguientes:

1) $\log$ LEG, $\log$ ILEG o $\log$ тот $=a_{0}+a_{1} \log U_{m x}^{*}+u_{1}$

2) $\log$ LEG, $\log$ ILEG O $\log$ TOT $=a_{0}+a_{2} \log \left(\stackrel{*}{\left(U^{m}\right.} / U u s\right)+u_{2}$

En donde: $U \stackrel{*}{*} x=$ series alternativas de la tasa de desempleo en México, y los símbolos restantes tienen el mismo significado que en el cuadro 4.

\section{BIBLIOGRAFIA}

Briggs, Vernon M., Jr., "Mexican Workers in the United States Labour Market: A Contemporary Dilemma", International Labour Review, CXII, Núm. 5, noviembre de 1975, pp. 351-368.

Browning, H., y W. Feindt, "Contexto social de la migración a Monterrey", en Movilidad social, migración y fecundidad en Monterrey metropolitano, México, Universidad de Nuevo León-University of Texas, 1967.

Campbell, Howard L., "Bracero Migration and the Mexican Economy, 1951-1964", tesis doctoral, The American University, 1972.

Copp, Nelson G., "Wetbacks' and Braceros: Mexican Migrant Laborers and American Immigration Policy, 1930-1960", tesis doctoral, Boston University, 1963.

Cornelius, Wayne A., y Juan Díez-Canedo, "Mexican Migration to the United States: the View from Rural Sending Communities", documento de investigación, inédito, Massachusetts Institute of Technology, 1976.

Fogel, Walter A., "Immigrant Mexicans and the U. S. Work Force", 
Monthly Labor Review, XCVIII, Núm. 5, mayo de 1975, pp. 44-46. Hernández Álvarez, José, "Perfil demográfico de la inmigración mexicana a los Estados Unidos, 1910-1950", DEMOGRAFía Y ECONOMÍA, Vol. 1, Núm. 1, 1967, pp. 18-39.

King, Jonathan, "Interstate Migration in Mexico", documento de investigación, inédito, Universidad de las Américas, sin fecha.

North, David S., y Marion F. Houstoun, "The Characteristics and Role of Illegal Aliens in the U. S. Labor Market", documento de investigación, inédito, Employment and Training Administration, U. S. Department of Labor, 1976.

Samora, Julian, Los mojados: The Wetback Story, Notre Dame, University of Notre Dame, 1971.

Unikel, Luis, Crescencio Ruiz Chiapetto y Omar Lazcano, "Factores de rechazo en la migración rural en México, 1950-1960", DEMOGRAFÍA Y ECONOMíA, Vol. VII, Núm. 1, 1973, pp. 24-57. 